\title{
Automation and Modernization of Meteorological Observation Network in Nigeria
}

\author{
HUSSAINI, A: YAKUBU, SO \\ ${ }^{* 1}$ Nigerian Meteorological Agency, Kaduna International Airport, Kaduna, Nigeria \\ ${ }^{2}$ Department of Mechanical Engineering, Nigerian Defence Academy, Kaduna, Nigeria \\ *Corresponding Author Email: usaini2000@yahoo.com,Tel: +2348033115536
}

\begin{abstract}
In Nigeria the beginning of automation of meteorological parameters was dated back to 1998 when five Automatic Weather Observation Systems (AWOSs) were installed at different locations. Unfortunately we were unable to sustain those equipment due to low literacy level of most operational staff available at the time of implementation. The Nigerian Meteorological Agency (NIMET) is making frantic efforts to ensure provision of timely and accurate Weather Information through acquisition of weather monitoring systems and training of technical and operational personnel on data capturing and data dissemination systems. In year 2006, NIMET embarked on installation of Automated Weather Observing Systems at four international airports namely Abuja, Lagos, Kano and Port Harcourt respectively. In 2009 additional Aviation Automated Weather Observing Systems (AWOS) at six other Airports such as Ilorin, Enugu, Owerri, Calabar, Katsina and Maiduguri were installed. Today, all synoptic stations in various location in Nigeria have AWOS installed.
\end{abstract}

\section{DOI: https://dx.doi.org/10.4314/jasem.v23i7.6}

Copyright: Copyright (C) 2019 Hussaini and Yakubu. This is an open access article distributed under the Creative Commons Attribution License (CCL), which permits unrestricted use, distribution, and reproduction in any medium, provided the original work is properly cited.

Dates: Received: 30 May 2019; Revised: 27 June 2019; Accepted 04 July 2019

Keywords: Automatic Weather Observation Systems, Environment, Nigerian Meteorological Agency, Observation

In line with the increasing needs of the developing world, it has become a necessity to obtain more reliable and continuous meteorological data and transfer these data in due course to those who are concerned. Today many sectors such as aviation, transportation, agriculture, construction, tourism, health, justice, security, national defense, written and visual press, and sports are very much in need of meteorological data support. Automatic Weather Observation Station (AWOS) was first experimented in Nigeria in 1998 when four numbers were installed in some of our airports namely Lagos, Abuja, Port Harcourt and Maiduguri. As at that time, Meteorological Observers were familiar with the manually observed instruments whose derived values are computed with slide rule and electronic calculator. Then, we had certain challenges which ranges from low level of computer literacy among observers to the fear of loss of jobs since fewer personnel would be required if automatic weather system is allowed to stay. Hence, data output from the newly introduced automated weather stations were never used to prepare weather forecasts and in short, the system did not see the light of the day. From the lesson learnt on the failure of the first set of Automatic weather stations, another set of AWOS was introduced in year 2000.This set of AWOS is solar powered and has a wireless telemetry whose console could be powered from two sources i.e. A.C. mains or battery. This type of AWOS is not dependent on the computer to retrieve data for the user because the data logger is right at the back of the receiving console. The only time a computer is needed is when data is to be downloaded from the logger to the computer for storage. This type of AWOS began to gain acceptance to the operators and we succeeded in installing about Forty Five numbers in our networks of stations. Since modernization of Meteorological Instrumentation has made it mandatory for both the operators and the maintenance personnel to have a good knowledge of end-user computing, the Nigerian Meteorological Agency organized computer appreciation training for its workforce and after this exercise, operators were no longer scare of computers and it became a useful tool that makes their jobs much easier. As of today, not less than thirty AWOS installed between year 2000 and 2005 are still functioning effectively.

The New Integrated Automated Weather Observing System: In year 2006, the Federal Government of Nigeria began a thorough overhauling of the Aviation sector and came up with the Safe Tower Project under which the four major international Airports were considered for the implementation of Aviation 
Integrated Weather Observing System. The system was successfully installed and commissioned in three of the four major airports, namely Lagos, Abuja and Port Harcourt. The installation at Kano Airport was delayed for the construction of the new control tower building to be completed. The parameters measured by this system are more than the conventional automatic weather station which provides Wind Speed, Wind Direction, Pressure, Temperature, Relative Humidity, Rainfall and Radiation values. The additional measurement from other sensors at the air side of the runway such as visibility, present weather and cloud base has made the system aviation AWOS. Having used the system for more than a couple of years, the Nigerian Meteorological Agency in year 2009, awarded contract for the procurement and installation of Aviation Weather Observing and Display System(AWODS) for six(6) Airports, namely Enugu, Owerri, Ilorin, Calabar, Katsina and Maiduguri Airports. Today, all airports and synoptic stations have AWOS installed and working. An automatic weather station (AWS) is defined as a "meteorological station at which observations are made and transmitted automatically" (WMO, 1992a). At an AWS, the instrument measurements are read out or received by a central data-acquisition unit. The collected data from the autonomous measuring devices can be processed locally at the AWS or elsewhere, for example, at the central processor of the network (WMO, 1989a). Automatic weather stations may be designed as an integrated concept of various measuring devices in combination with the data-acquisition and processing units. Such a combined system of instruments, interfaces and processing and transmission units is usually called an automated weather observing system (AWOS) or automated surface observing system (ASOS). Automatic weather stations are used for increasing the number and reliability of surface observations.

Why AWOS: (a) Increasing the density of an existing network by providing data from new sites and from sites that are difficult to access and inhospitable; (b) Supplying, for manned stations, data outside the normal working hours; (c) Increasing the reliability of measurements by using sophisticated technology and modern, digital measurement techniques; (d) Ensuring the homogeneity of networks by standardizing the measuring techniques; (e) Satisfying new observational needs and requirements; (f) Reducing human errors; (g) Lowering operational costs by reducing the number of observers; (h) Measuring and reporting with high frequency or continuously.

\section{Development and Installation of AWOS}

Meteorological requirements: The development and installation of AWOSs should be the result of a definite, coordinated plan for getting data to users in the format required. The Commission for Instruments and Methods of Observation (CIMO) gives the following advice to Members of WMO and, by inference, to any Service taking meteorological measurements. When considering the introduction of new AW0S instrument systems, Meteorological Services should: (a) Introduce into service only those systems that are sufficiently well documented so as to provide adequate knowledge and understanding of their capabilities, characteristics and any algorithms used; (b) Retain or develop sufficient technical expertise to enable them to specify system requirements and to assess the appropriateness of the capabilities and characteristics of such systems and algorithms used therein; (c) Explore fully user requirements and engage users in system design of AWOSs; (d) Engage users in validation and evaluation of the new automated systems; (e) Engage manufacturers in the system assessment and need for improvements in performance; (f) Develop detailed guides and documentation on the systems to support all users; (g) Develop adequate programmes for maintenance and calibration support of the AWOSs; (h) Consult and cooperate with users, such as aeronautical authorities, throughout the process from AWS design, to implementation, to operational use; (i) Develop and apply reporting methods for national use to accommodate both observations generated by traditional and automated systems. With respect to the automation of traditional visual and subjective observations, and future changes in reporting code, Meteorological Services should improve their definition of requirements with respect to: (a) Areas of application for which data are no longer required; (b) Areas of application for which different or new data are needed; (c) Prioritizing the requirements for data to be provided by AWOSs. When considering the development and application of algorithms for AWOSs, Meteorological Services should: (a) Encourage instrument and system designers to work closely with relevant users to understand fully user requirements and concerns; (b) Work together with system designers to publish and disseminate, for widespread use and possible standardization, descriptions of the data-processing algorithms used in their systems to derive meteorological variables; (c) Test and evaluate thoroughly new algorithms and systems being introduced and disseminate the test results in the form of performance characteristics to users of the observations; (d) Evaluate thoroughly, through field testing and intercomparison, the relationship of new algorithms and systems to previous methods, and establish transfer functions for 
use in providing data continuity and homogeneity, and disseminate these data to users.

Climatological Requirements: Where a proposed automatic station has a role in providing data for climatological records, it is important for the integrity, homogeneity and utility of the climate data sets that the following areas be considered for action (WMO, 1993): (a) In cases where an AWOS replaces a manual observing system that has been in operation for a long time, a sufficient overlap in observation systems to facilitate maintaining the homogeneity of the historical record must be assured. The overlap time is dependent on the different measured variables and on the climate region. In tropical regions and islands, the overlap time could be shorter than in extra tropical and mountainous regions. The following general guidelines are suggested for a sufficient operational overlap between existing and new automated systems: (i) Wind speed and direction: 12 months (ii) Temperature, humidity, and sunshine, evaporation: 2 months (iii) Precipitation: 0 months (It will often be advantageous to have an ombrometer operated in parallel with the automatic rain-gauge). A useful compromise would be an overlap period of 2 months (i.e. two seasonal cycles); (b) accurate metadata should be maintained for each AWOS installation; (c) Procedures should be standardized for quality assurance and processing of data from AWOSs (d) The existing and future requirements of climate data users should be defined precisely and considered in developing statements of requirement for automated observations by AWOSs; (e) Climate users should be trained in the most effective use of AWOS data;(f) Specifications for a standardized climatological AWS should be developed which would record a basic set of climate variables such as temperature, precipitation, pressure and wind. Standardized water vapour measurements should be included due to the significance of this parameter in climate-change studies. Extreme values of all variables should be accurately and consistently recorded in a way that can be precisely related to older, manually-observed, data.

Siting and Exposure: Site selection for meteorological observing stations are designed so that representative measurements (or observations) can be taken according to the type of station involved. Thus, a station in the synoptic network should make observations to meet synoptic-scale requirements, whereas an aviation meteorological observing station should make observations that describe the conditions specific to the local (aerodrome) site. Where stations are used for several purposes, for example, aviation, synoptic and climatological purposes, the most stringent requirement will dictate the precise location of an observing site and its associated sensors. A detailed study on siting and exposure is published in WMO (1993a).

As an example, the following considerations apply to the selection of site and instrument exposure requirements for a typical synoptic or climatological station in a regional or national network: (a) Outdoor instruments should be installed on a level piece of ground, preferably no smaller than $25 \mathrm{~m}$ x $25 \mathrm{~m}$ where there are many installations, but in cases where there are relatively few installation, the area may be considerably smaller, for example, $10 \mathrm{~m} \times 7 \mathrm{~m}$ (the enclosure). The ground should be covered with short grass or a surface representative of the locality, and surrounded by open fencing or palings to exclude unauthorized persons. Within the enclosure, a bare patch of ground of about $2 \mathrm{~m} \times 2 \mathrm{~m}$ is reserved for observations of the state of the ground and of soil temperature at depths of equal to or less than $20 \mathrm{~cm}$ (soil temperatures at depths greater than $20 \mathrm{~cm}$ can be measured outside this bare patch of ground). (b) There should be no steeply sloping ground in the vicinity, and the site should not be in a hollow. If these conditions are not met, the observations may show peculiarities of entirely local significance; (c) The site should be well away from trees, buildings, walls or other obstructions. The distance of any such obstacle (including fencing) from the raingauge should not be less than twice the height of the object above the rim of the gauge, and preferably four times the height; (d) The sunshine recorder, raingauge and anemometer must be exposed according to their requirements, preferably on the same site as the other instruments; (e) It should be noted that the enclosure may not be the best place from which to estimate the wind speed and direction; another observing point, more exposed to the wind, may be desirable; (f) Very open sites which are satisfactory for most instruments are unsuitable for raingauges. For such sites, the rainfall catch is reduced in conditions other than light winds and some degree of shelter is needed; (g) If in the instrument enclosure surroundings, maybe at some distance, objects like trees or buildings obstruct the horizon significantly, alternative viewpoints should be selected for observations of sunshine or radiation;(h) The position used for observing cloud and visibility should be as open as possible and command the widest possible view of the sky and the surrounding country; (i) At coastal stations, it is desirable that the station command a view of the open sea. However, the station should not be too near the edge of a cliff because the wind eddies created by the cliff will affect the wind and precipitation measurements; (j) Night observations of cloud and visibility are best made from a site unaffected by extraneous lighting. 
Coordinates of the Station: The position of a station referred to in the World Geodetic System 1984 (WGS84) Earth Geodetic Model 1996 (EGM96) must be accurately known and recorded.

The coordinates of a station are: (a) The latitude (b) The longitude (c) The height of the station above mean sea level, the elevation of the station, to the nearest metre.

These coordinates refer to the plot on which the observations are taken and may not be the same as those of the town, village or airfield after which the station is named. The elevation of the station is defined as the height above mean sea level of the ground on which the raingauge stands or, if there is no raingauge, the ground beneath the thermometer screen. If there is neither raingauge nor screen, it is the average level of terrain in the vicinity of the station. If the station reports pressure, the elevation to which the station pressure relates

Changes of Instrumentation and Homogeneity: The characteristics of an observing site will generally change over time, for example, through the growth of trees or erection of buildings on adjacent plots. Sites should be chosen to minimize these effects, if possible. Documentation of the geography of the site and its exposure should be kept and regularly updated as a component of the metadata (WMO, 2003b). It is especially important to minimize the effects of changes of instrument and/or changes in the siting of specific instruments. Although the static characteristics of new instruments might be well understood, when they are deployed operationally they can introduce apparent changes in site climatology. In order to guard against this eventuality, observations from new instruments should be compared over an extended interval (at least one year; see the Guide to Climatological Practices (WMO, 1993) before the old measurement system is taken out of service. The same applies when there has been a change of site. Where this procedure is impractical at all sites, it is essential to carry out comparisons at selected representative sites to attempt to deduce changes in measurement data which might be a result of changing technology or enforced site changes.

\section{INSPECTION AND MAINTENANCE}

Inspection of Stations: All synoptic land stations and principal climatological stations should be inspected no less than once every two years. Agricultural meteorological and special stations should be inspected at intervals sufficiently short to ensure the maintenance of a high standard of observations and the correct functioning of instruments. The principal objective of such inspections is to ascertain that: (a)
The siting and exposure of instruments are known, acceptable and adequately documented; (b) Instruments are of the approved type, in good order, and regularly verified against standards, as necessary; (c) There is uniformity in the methods of observation and the procedures for calculating derived quantities from the observations; (d) The observers are competent to carry out their duties; (e) The metadata information is up to date.

Maintenance: Observing sites and instruments should be maintained regularly so that the quality of observations does not deteriorate significantly between station inspections. Routine (preventive) maintenance schedules include regular "housekeeping" at observing sites (for example, grass cutting and cleaning of exposed instrument surfaces) and manufacturers' recommended checks on automatic instruments. Routine quality control checks carried out at the station or at a central point should be designed to detect equipment faults at the earliest possible stage. Depending on the nature of the fault and the type of station, the equipment should be replaced or repaired according to agreed priorities and timescales. As part of the metadata, it is especially important that a $\log$ be kept of instrument faults, exposure changes, and remedial action taken where data are used for climatological purposes, WMO (1989).

\section{MAINTENANCE PROCEDURE}

Maintenance at AWOS sites and at Base Stations may be done as follows and as the situation from the sensors or the workstation demands:

* Inspect s the whole system

* Clean cloud height, Day/Night, Visibility

Sensor, and Present Weather Sensor optics

* Remove debris from system

* Check for mechanical damage

* Check for movement of wind speed and direction sensors

* Check obstruction lights

* Check display operation

* Inspect data collection Platform

* Check sensor interface processor

* Check sensor and mast for damage/corrosion

* Check cables and hardware connections

* Clean all surfaces

* Check Batteries

* Check Alignment of sensors where applicable

In general, it is not advisable to repair AWOS sensors or other modules in the field because conditions do not favour effective work. Also, because of high staff costs and relatively low equipment costs, it is more 
cost-effective to discard faulty modules rather than to repair them.

Types of Automatic Weather Stations: A general classification could include stations that provide data in real time and those that record data for non-realtime or off-line analysis. It is not unusual, however, for both of these functions to be discharged by the same AWOS.

Real-time AWOS: A station providing data to users of meteorological observations in real time, typically at programmed times, but also in emergency conditions or upon external request. Typical real-time use of an AWOS is the provision of synoptic data and the monitoring of critical warning states such as storms and river or tide levels.

Off-line AWOS: A station recording data on site on internal or external data storage devices possibly combined with a display of actual data. The intervention of an observer is required to send stored data to the remote data user. Typical stations are climatological and simple aid-to-the-observer stations. Since AWOSs can be very expensive, the stations' facilities can also be used to satisfy the common and specific needs and requirements of several applications, such as synoptic, aeronautical and agricultural meteorology, hydrology and climatology. They may also be used for special purposes, such as nuclear power safety, air and water quality, and road meteorology. Some AWOSs are, therefore, multipurpose AWOSs.

Networking: An AWOS usually forms part of a network of meteorological stations, each transmitting its processed data to a central network processing system by various data transmission means. As the tasks to be executed by this central system are strongly related, and often complementary, to the tasks of the AWOSs, the functional and technical requirements of both the central system and the AWOSs should be very well coordinated. When planning the installation and operation of a network of AWOSs, it is of the utmost importance to consider the various problems associated with maintenance and calibration facilities, their organization and the training and education of technical staff.

The layout of an AWOS typically consists of the following: (a) On a standard observing area, preferably no smaller than $25 \mathrm{~m}$ x $25 \mathrm{~m}$ (WMO, 1989a), a series of automated sensors sited at the recommended positions and interconnected to one or more data collection units using interfaces, or for an AWOS, a set of sensors installed in close combination, but not affecting each other, directly connected to a central processing unit (CPU) by means of shielded cables, fibre optics, or radio links; (b) A CPU for sensor data-acquisition and conversion into a computer-readable format, proper processing of data by means of a microprocessor-based system in accordance with specified algorithms, the temporary storage of processed data, and their transmission to remote users of meteorological information; (c) Peripheral equipment such as a stabilized power supply providing power to the various parts of the station, a real-time clock, and built-in test equipment for automatic monitoring of the status of vital parts of the station.

Central processing unit: The core of an AWOS is its CPU. Its hardware configuration depends on the complexity and magnitude of the functions it has to perform and on whether a unique hardware solution exists. In general, the main functions of the CPU are data acquisition, data processing, data storage and transmission. Depending on local circumstances and requirements, the different functions of the CPU may also be executed by different units. In such cases, each unit has its own microprocessor and relevant software, can be installed at different places in the station, and can communicate with each other through wellestablished inter-processor data transfer links and procedures. They operate in a dependency relation, the data-processing unit being the independent unit. The rapid technological evolution of modern industrial data-acquisition and process-control systems opens up new possibilities for meteorological applications. The high degree of input/output modulation and flexibility, the drastically increased operating speed of microprocessors and, in particular, the availability of dedicated data-acquisition, process control and telecommunications software make it possible to develop AWOSs which can meet the diverse observation needs and requirements of various users.

Data acquisition: In general, the data-acquisition hardware is composed of (a) Signal-conditioning hardware for preventing unwanted external sources of interference from influencing the raw sensor signals, for protecting the CPU electronics, and for adapting signals to make them suitable for further data processing; (b) Data-acquisition electronics with analogue and digital input channels and ports, scanning equipment and data conversion equipment to enter the signals into the CPU memory.

Signal conditioning: Signal conditioning is a vital function in the data acquisition process and starts with the proper selection of cables and connectors for connecting the sensor to the data-acquisition 
electronics. It is further accomplished by means of different hardware modules. Taken over from industrial process control, several conditioning functions are now integrated into one removable module. The most convenient and, hence, most common location for installing these modules is on the terminal panels of sensor cables in the same waterproof enclosure as the data-acquisition electronics. Depending on the sensor and local circumstances, various signal-conditioning techniques are available.

Sensor cables: Electrical signals from the sensors entering a data-acquisition system might include unwanted noise. Whether this noise is troublesome depends upon the signal-to-noise ratio and the specific application. Digital signals are relatively immune to noise because of their discrete (and high-level) nature. In contrast, analogue signals are directly influenced by relatively low-level disturbances. The major noise transfer mechanisms include capacitive and inductive coupling. A method of reducing errors due to capacitive coupling is to employ shielded cables for which a conductive material (at ground potential) is placed between the signal cables and the interference source. The additional use of a pair of wires that are entwined is effective in reducing electromagnetic coupling.

Surge protection: When an AWOS could be subject to unintentional high-voltage inputs, the installation of a protection mechanism is indispensable to avoid possible destruction of the equipment. High-voltage input can be induced from magnetic fields, static electricity and, especially, from lightning.

Two-wire transmitters: It is sometimes desirable to pre-amplify low-level signals close to the sensor to maintain a maximum signal-to-noise ratio. One way of performing this kind of signal conditioning is to use a two-wire transmitter. These transmitters not only amplify the input signal, but also provide isolation and conversion to a high-current level (typically to 20 $\mathrm{mA}$ ).

Amplifiers: Analogue sensor signals can vary in amplitude over a wide range. The analogue-to-digital (A/D) converter, however, requires a high-level signal in order to perform best. In many cases, an amplifier module is used to boost possible low-level signals to the desired amplitude.

Resistances: Special modules are used to convert resistances, such as platinum thermometers, into a linearized output voltage signal and to provide the necessary output current for this conversion. It should be noted that the conversion to a linear signal can introduce inaccuracies, which can be critical for some applications.

Data Processing: The data-processing hardware is the heart of the CPU and its main functions are to act as the master control of the input/output of data to, and from, the CPU and to carry out the proper processing of all incoming data by means of the relevant software. The hardware is operated by a microprocessor. Microprocessors do not change the principles of meteorological measurements or observing practices but they do allow the instrument designer to perform technical functions in a new way to make measurements easier, faster and more reliable, and to provide the instrument with higher capabilities, especially in data handling. The adoption of microprocessors considerably reduces hardware costs for some applications. It must be noted, however, that the expanded expectations which may be met by this device will lead very often to a fast-growing and considerably underestimated cost of the development of software.

Data Transmission: The data transmission part of the CPU forms the link with the "outside world", which may be the local observer or the maintenance personnel, the central network processing system of the National Meteorological and Hydrological Service, or even directly the users of meteorological information. The equipment is interfaced to the CPU by using commonly available serial and parallel input/output ports. The most suitable means of data transmission depends mainly on the site in question and the readily available transmission equipment. No single solution can be regarded as universally superior, and sometimes the transmission chain requires the use of several means. The conversion of raw sensor data consists of the transformation of the electrical output values of sensors or signal-conditioning modules into meteorological units. The process involves the application of conversion algorithms making use of constants and relations obtained during calibration procedures. An important consideration is that some sensors are inherently non-linear, namely their outputs are not directly proportional to the measured atmospheric variables (for example, a resistance thermometer), that some measurements are influenced by external variables in a non-linear relation (for example, some pressure and humidity sensors are influenced by the temperature) and that, although the sensor itself may be linear or incorporate linearization circuits, the variables measured are not linearly related to the atmospheric variable of interest (for example, the output of a rotating beam ceilometer with photo detector and shaft-angle encoder providing 
backscattered light intensity as a function of angle is nonlinear in cloud height). As a consequence, it is necessary to include corrections for non-linearity in the conversion algorithms as far as this is not already done by signal-conditioning modules. Linearization is of particular importance when mean values must be calculated over a certain time. Indeed, when the sensor signal is not constant throughout the averaging period, the "average then linearize"

Consistent Improvement of AWOSs: The Nigerian Meteorological Agency has a check list for the continual improvement of the AWOSs, the checklist has some of the following questions:

Do you have AWOS?

If yes, what type do you have?

Type of parameters measured

Did you receive any formal training?

What type of training did you received?

How and where did you receive the training?

Is the AWOS SERVICEABLE?

If not serviceable, what are the challenges?

When was the AWOS INSTALLED?

How do you Archive and transmit the data

Conclusion: An automatic weather station (AWOS) is an automated version of the traditional weather station, either to save human labour or to enable measurements from remote areas. An AWS will typically consist of a weather-proof enclosure containing the data logger, rechargeable battery, and the meteorological sensors with an attached solar panel or wind turbine and mounted upon a mast (usually $10 \mathrm{~m}$ in height). The specific configuration may vary due to the purpose of the system. The system may report in near real time or save the data for later recovery. In the past, automatic weather stations were often placed where electricity and communication lines were available. Nowadays, the solar panel, wind turbine and mobile phone technology have made it possible to have wireless stations that are not connected to the electrical grid or hardline telecommunications network. The application of AWOS is broad in all Engineering fields, Agriculture, Water Resources Management, Aviation, Military and Security.

\section{REFERENCES}

World Meteorological Organization (1983). Guide on the Global Data-processing System. WMO-No. 305, Geneva.

World Meteorological Organization (1989). Preliminary results of the WMO automatic sunshine duration measurement comparison 1988/89 in Hamburg (K. Dehne). Fourth WMO Technical Conference on Instruments and Methods of Observation (TECIMO-IV). Instruments and Observing Methods Report No. 35, WMO/ TD-No. 303, Geneva.

World Meteorological Organization (1989b). WMO International Hygrometer Intercomparison ( $\mathrm{J}$. Skaar, K. Hegg, T. Moe and K. Smedstud). Instruments and Observing Methods Report No. 38, WMO/ TD-No. 31, Geneva.

World Meteorological Organization (1990a). The First WMO Intercomparison of Visibility Measurements (United Kingdom 1988/1989) (D.J. Griggs, D.W. Jones, M. Ouldringe and W.R. Sparks). Instruments and Observing Methods Report No. 1, WMO/TD-No. 01, Geneva.

World Meteorological Organization, (1992a). International Meteorological Vocabulary. Second edition, WMO-No. 182, Geneva.

World Meteorological Organization (1992b). The WMO Automatic Digital Barometer Intercomparison: Final Report (J.P. van der Meulen). Instruments and Observing Methods Report No., WMO/TD-No., and Geneva.

World Meteorological Organization (1993). Guide on Meteorological Observation and Information Distribution Systems at Aerodromes. WMO-No. 31, Geneva.

World Meteorological Organization (2003b). Algorithms Used in Automatic Weather Stations: Evaluation of Questionnaire (M.D. Gifford). Instruments and Observing Methods Report No. 8, WMO/TD-No. 110, Geneva. 